\title{
The Gravity Model Specification for Modeling International Trade Flows and Free Trade Agreement Effects: A 10-Year Review of Empirical Studies
}

\author{
Konstantinos Kepaptsoglou*, Matthew G. Karlaftis and Dimitrios Tsamboulas
}

Department of Transportation Planning and Engineering, School of Civil Engineering, National Technical University of Athens, 5 Iroon Polytechniou Str., Athens, 15773, Greece

\begin{abstract}
The gravity model has been extensively used in international trade research for the last 40 years because of its considerable empirical robustness and explanatory power. Since their introduction in the 1960's, gravity models have been used for assessing trade policy implications and, particularly recently, for analyzing the effects of Free Trade Agreements on international trade. The objective of this paper is to review the recent empirical literature on gravity models, highlight best practices and provide an overview of Free Trade Agreement effects on international trade as reported by relevant gravity model-based studies over the past decade.
\end{abstract}

Keywords: Gravity model, review, trade flows, free trade agreements, models, econometric methods.

\section{INTRODUCTION}

Since its introduction by Tinbergen [1] and Linneman [2], the gravity model has been widely used for explaining flows of international trade. The gravity specification has exhibited considerable empirical robustness and explanatory power for describing trade flows [3,4], despite its lack of strong theoretical foundation [3]. As reported by Porojan [4], in the last decade gravity models have been employed in numerous studies for analyzing and assessing trade flows. Indeed, the empirical literature reveals a considerable number of publications offering either methodological advancements or refinements, or attempting to explain policy impacts on trade flows.

A particular application of the gravity model is to explain and predict the effects of Free Trade Agreements on trade flows [5]. Free Trade Agreements are forms of trade pacts between countries; these agreements eliminate tariffs, quotas and other barriers for a number of goods (if not for all), traded between involved partners. The aim of FTAs is obvious: increased trade between two countries as a result of relaxing or removing existing institutional and economic barriers. FTAs have been a tool widely implemented for enhancing trade between countries; examples include the North American Free Trade Agreement between the USA, Canada and Mexico (NAFTA), the Free Trade Agreement of the Americas (FTAA), the ASEAN FTA between eastern Asian countries and the Euro-Mediterranean Free Trade Agreement (EMFTA).

The research community has expressed a wide interest in the aspects of the gravity specification and its application in

\footnotetext{
*Address correspondence to this author at the Department of Transportation Planning and Engineering, School of Civil Engineering, National Technical University of Athens, 5 Iroon Polytechniou Str., Athens, 15773, Greece; Tel: +30-210-7721280; Fax: +30-2107722404;

E-mail: kkepap@central.ntua.gr
}

representing trade flows and particularly FTA effects. In that context, this paper provides a systematic review of recent work on trade flow modeling from an empirical perspective and offers a basis for evaluating current research. A secondary objective is to provide insights on the effects of FTAs on trade as explained by gravity model specifications. The remainder of the paper is structured as follows: the next section includes an overview of the different modeling approaches for explaining trade flows. Then, the gravity model is briefly presented and a framework for categorizing existing studies is proposed. Based on that framework, studies undertaken in the last decade (1999-2009) are reviewed and critically discussed. Additionally, a short analysis of FTA modeling issues and empirical findings of effects is provided. The final section summarizes the conclusions of the paper.

\section{OVERVIEW OF TRADE FLOW MODELING: ECONOMETRICS VERSUS SIMULATION}

Trade flow modeling has been widely researched in the last three decades; most efforts for expressing and analyzing trade flows have concentrated (a) on simulation models that aim at replicating that phenomenon and its impacts, and (b) on econometric models that attempt to make predictions based on past, actual performance [6].

Simulation models capture the underlying structure of trade flows (comprising of activities such as production, consumption and transportation) [7], respond to inputs and estimate potential impacts to trade flows. Frequently, inputoutput (I/O) and generalized equilibrium models have been exploited for simulating trade flows with the later having the advantage of calculating trade flows endogenously and incorporating transportation costs in the model [7]. In particular, computable generalized equilibrium (CGE) models have been widely applied for modeling trade flows and examining FTA effects [8-15]. However, CGE models 
of trade have been criticized by a number of researchers; for instance, the World Bank Economic Prospects of 2005 [6] note that CGE models require the selection of a considerable number of parameters; since these parameters are chosen and not estimated, statistical properties of the results are unknown. Also, Hertel et al. [16] comment on the weak econometric foundations of CGEs, which is indicated by past research; they find however that some of the results of CGEs are robust (especially those related to the analysis of FTA effects) and conclude that there is a good potential of combining CGEs with econometric models for obtaining better results.

Econometric approaches for modeling trade flows have focused on the gravity model specification. The concept of the gravity model is based on Newtonian physics; trade between two partners is affected by their sizes and proximity $[4,17]$. In particular, a flow of goods between two areas is expressed as a function of the characteristics of the origin and of the destination and of some measure of impedance between them. The gravity model has demonstrated an excellent empirical robustness in describing trade flows, despite its often asserted lack of theoretical background and poor reputation among economists [18]. For example, Bergstrad [3] reported that, "despite the model's consistently high statistical explanatory power, its use for predictive purposes has been inhibited owing to an absence of strong theoretical foundations". Moreover, as noted by Filipinni and Molini [19], while the gravity model has been often characterized as "facts without theory", consistency of its results with facts makes it very popular for practical applications.

Indeed, early justifications on the gravity model performance were provided by Linnermann [2] while Anderson [20] was the first to derive a theoretical explanation based on economic theory. Krugman [21], Helpman and Krugman [22], Bergstrad [3,23], Deardorff [24] and Anderson and Mercouiller [25] presented alternative theoretical foundations for the gravity model and Baldwin [18] noted that, "in contrast to popular belief it (the gravity model) does have such foundations". However, since a discussion of the gravity model theoretical derivation is not within the scope of the paper, the reader is referred to Baier and Bergstrad [26], Evennet and Keller [27] and Filippini and Molini [19] for related reviews.

Both simulation and econometric approaches have been exploited in an effort to capture the effects on various factors on trade flows; however the gravity model is the most popular among them, mainly because of its robust performance and limited need for parameter assumptions [19]. As a result, numerous studies on gravity models and their application in trade flows have emerged during the last decade and are discussed in the following sections.

\section{THE GRAVITY MODEL SPECIFICATION}

Consider a flow of goods $F_{i j}$ between two areas $i$ and $j$. $F_{i j}$ can then be expressed as a function of the characteristics of the origin $\left(O_{i}\right)$ and the destination $\left(D_{j}\right)$ areas and some measure of impedance between them $\left(R_{i j}\right)$ :
$F_{i j}=O_{i} \cdot D_{j} \cdot R_{i j}$

Equation (1) can be modeled as a linear function by taking its logs:

$F^{\prime}{ }_{i j}=\log F_{i j}=\beta \cdot X+\varepsilon, \varepsilon \sim \mathrm{N}\left(0, \sigma^{2}\right)$

where

$X$ : Vector containing the logs of explanatory variables

$\boldsymbol{\beta}: \quad$ Vector of parameters to be estimated

$\varepsilon: \quad$ Error term

Equation (2) expresses the relation between trade flows (which can be bilateral, imports or exports) and explanatory variables which refer to the sizes of the trading partners' economies (mass variables), their proximity and any other factors promoting or discouraging trade between them (impedance variables). Anderson and van Wincoop [28] provide an excellent discussion on the theoretical background behind the derivation of equation (2); which is, as noted earlier, out of the scope of this paper.

The gravity model often serves as a practical tool for estimating trade flows and examining explanatory factors and policy implications on them; the latter could include the potential of adding novel parameters and factors to the model or investigating the effects of already known parameters to trade flows. Moreover, the statistical techniques applied could result to more or less accurate model parameters [29]. Considering these, a three layer framework (Fig. 1) is proposed for organizing studies related to gravity based trade flow modeling (objectives and applications, parameters, and methodology). The "objectives and applications" layer focuses on the scope of each study; whether the gravity model development is the target or the model serves as the tool for investigating policies related to trade flows. The "Parameters" layer incorporates all items necessary for developing the model; namely explanatory variables and the type and size of dataset. As for the "Methodology layer", this incorporates the variety of statistical and econometric techniques used for estimating the parameters of the gravity model specification.

\subsection{Objectives and Applications: Target or Tool?}

Gravity models are commonly used to investigate trade flows and related policies (Table $\mathbf{1}$ includes most recent research in this area along with some of the most important findings of each). For instance, some studies analyze trade flows between regions in general $[19,30-36]$ or flows of specific products [32, 37, 38]. However, most recent studies focus on examining the effects of regional trade agreements, currency unions and common markets and particularly whether these result in creating or diverting trade; in this research area see, for example [5, 31, 38-64]. Other researchers have examined trade policy implications and factors that affect trade, such as natural border effects [65], monetary union impacts [66, 67], domino effects [68], the foreign direct investments [69], the rules-of-origin [70], transportation costs [71, 72], neutral partners theory [68], trade union rights and democracy effects [73], trade 


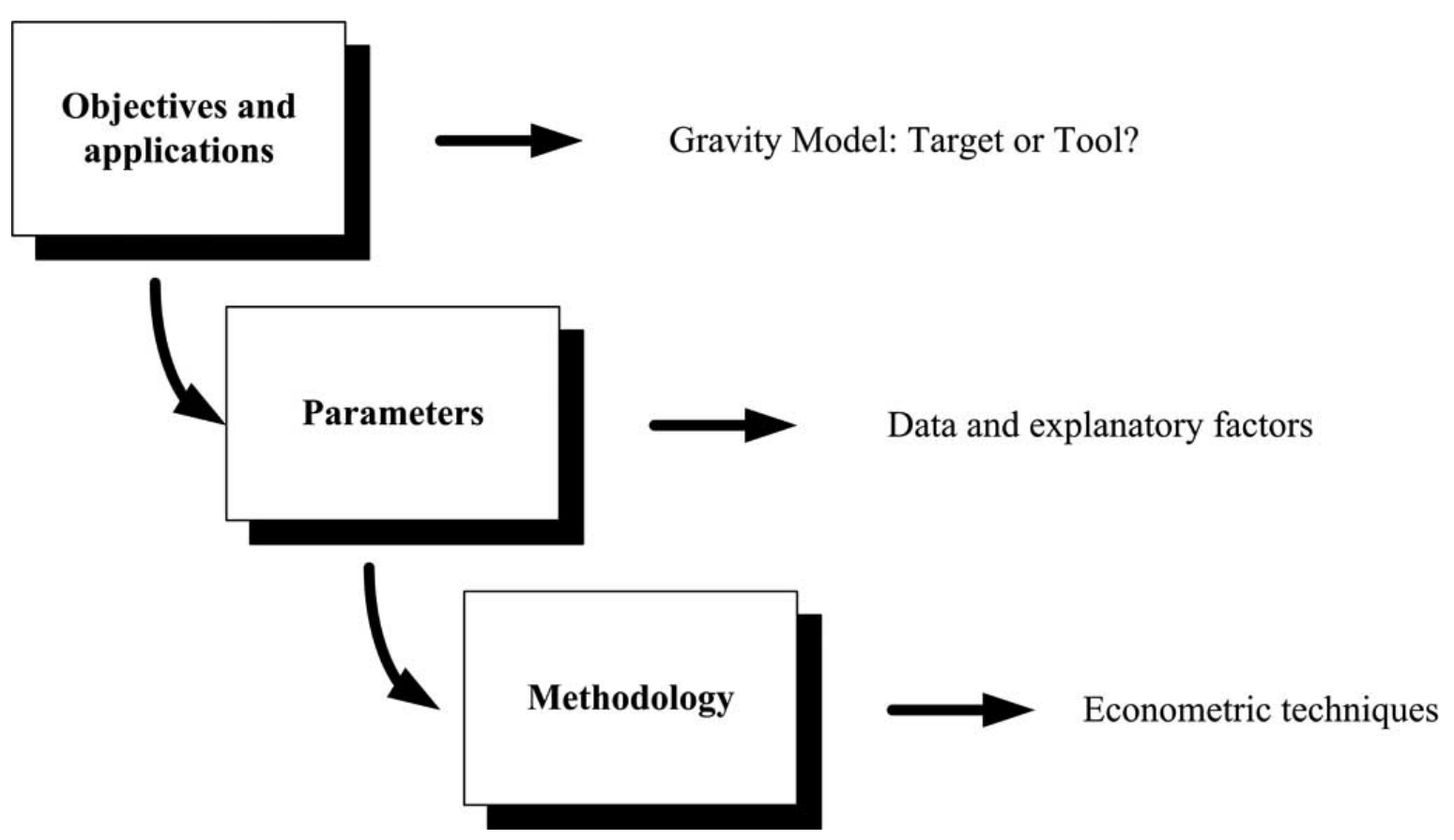

Fig. (1). Review framework and layers.

facilitation, regulatory quality and export performance [74] and north versus south effects when controlling for distance [75].

Apart from analyzing policies and flows, a number of studies have attempted to improve the performance of gravity models and aimed at introducing methodological novelties in estimating their parameters. Such cases include the incorporation of spatial effects and stochastic aspects to the gravity model and the application of novel econometric methods for the estimation of its parameters $[4,5,57,76-$ 82]. However, most studies reviewed do offer methodological novelties although this is not their main objective.

\subsection{Parameters: Datasets and Variables}

The "parameters" incorporates both the factors and explanatory variables used in the reviewed papers, as well as the dataset types and characteristics exploited for developing the models.

\subsubsection{Datasets}

Panel data are used in most studies for periods of at least 5 years; only few recent studies draw conclusions based on cross sectional data based on a single year or an average of a period. Matyas [83] noted that bilateral trade flows are naturally represented through a three way specification which includes time, exporter and importer characteristics. Therefore, excluding an important source of variation such as time, could lead to inconsistent modeling results. Ghosh and Yamarik [84] showed that gravity models based on cross-sectional data yield unstable results. Moreover, according to Nowak-Lehmann et al. [58], panel data offer several advantages such as the possibility of capturing relationships over variables in time and observing individual effects between trading partners.

\subsubsection{Dependent and Explanatory Variables}

Exports and bilateral trade flows are the most common dependent variables found in trade flow gravity models, while their explanatory variables can be distinguished in the following two groups:

$>$ Factors indicating demand and supply of trading countries,

$>$ Factors representing the impedance imposed on a trade flow between countries.

Common proxies for demand and supply are measures of a country's economic and market size; income level, population, area size and GDP per capita are variables included in most gravity model specifications. In particular, GDP per capita indicates the purchase power of importing and exporting countries [33]; two countries with considerably different populations may have similar GDPs but totally different economic development. According to Bergstrad [3], many studies explicitly consider GDP and GDP per capita as explanatory variables, with GDP per capita serving as a proxy for the capital - endowment ratio [3]. Furthermore, some specifications alternatively incorporated size similarity, originally motivated by Helpman [85], which is a function of the partnering countries' GDPs (for instance, Baltagi et al. [77]).

In addition to the above, some studies exploited total imports and exports along with those aforementioned factors; imports and exports are considered as more 'direct' indicators of a country's demand and supply characteristics [3]. Import and export volumes may be considered directly (see, for example, Fukao et al. [43]; Musila [50]; Kepaptsoglou et al. [64]), even as a lagged variable (exports of the previous time period - Filippini and Molini [19]). Studies by Peridy [53] and Sohn [33] expressed these 
Table 1. Empirical Studies on International Trade Modeling, 1999-2009

\begin{tabular}{|c|c|c|c|c|c|c|}
\hline Year & Authors & Objective & Dataset & $\begin{array}{l}\text { Dependent } \\
\text { Variables }\end{array}$ & $\begin{array}{l}\text { Explanatory } \\
\text { Variables }\end{array}$ & $\begin{array}{l}\text { Estimation } \\
\text { Technique }\end{array}$ \\
\hline 1999 & Kalirajan & $\begin{array}{c}\text { Incorporation of stochastic } \\
\text { aspects in the gravity } \\
\text { model coefficients }\end{array}$ & $\begin{array}{l}\text { Panel Data, Australia and } \\
\text { Indian Ocean rim trading } \\
\text { partners, 1990-1994 }\end{array}$ & Exports & GDP, GDP per capita, distance & $\begin{array}{l}\text { Stochastic } \\
\text { Varying } \\
\text { Coefficients } \\
\text { model }\end{array}$ \\
\hline 1999 & Endoh & $\begin{array}{c}\text { Investigation of trade } \\
\text { creation and diversion in } \\
\text { the EEC, LAFTA and } \\
\text { CMEA }\end{array}$ & $\begin{array}{l}\text { Panel Data, EEC, LAFTA and } \\
\text { CMEA members, 1960-1994 }\end{array}$ & Exports & $\begin{array}{c}\text { GDPs, population, distance, } \\
\text { common language, intra- } \\
\text { member, inter-member trade and } \\
\text { trade with non-members }\end{array}$ & OLS \\
\hline 1999 & Breuss and Egger & $\begin{array}{l}\text { Examination of East - } \\
\text { West Europe trade } \\
\text { potentials }\end{array}$ & $\begin{array}{l}\text { Cross sectional data, old (24) } \\
\text { OECD countries, averages of } \\
\text { the period } 1990-1994\end{array}$ & Exports & $\begin{array}{c}\text { GDPs per capita, population, } \\
\text { distance, common language, } \\
\text { EU12 and NAFTA memberships }\end{array}$ & OLS \\
\hline 2000 & Rose & $\begin{array}{l}\text { Analysis of common } \\
\text { market effects on trade }\end{array}$ & $\begin{array}{c}\text { Panel data, } 186 \text { countries, } \\
1970,1975,1980,1985,1990\end{array}$ & Exports & $\begin{array}{l}\text { GDP, GDP per capita, distance, } \\
\text { common border, common } \\
\text { language, FTA, common nation, } \\
\text { colony, common currency, } \\
\text { bilateral exchange rate }\end{array}$ & OLS \\
\hline 2000 & Arghyriou & $\begin{array}{c}\text { Investigation of effects in } \\
\text { trade by Greece's } \\
\text { participation in the EU }\end{array}$ & $\begin{array}{c}\text { Panel data, Greece and major } \\
\text { trade partners, Averages } \\
1970-1980,1981-1992\end{array}$ & $\begin{array}{l}\text { Imports and } \\
\text { Exports }\end{array}$ & $\begin{array}{c}\text { GDP, Pre-Post integration period } \\
\text { in the EU, exchange rate, } \\
\text { monetary policy }\end{array}$ & OLS \\
\hline 2000 & Nitsch & $\begin{array}{l}\text { Investigation of natural } \\
\text { border effect in trade in } \\
\text { the EU }\end{array}$ & $\begin{array}{c}\text { Panel data, EU-12 countries, } \\
1979-1990\end{array}$ & Exports & $\begin{array}{c}\text { GDP, distance, common border, } \\
\text { common language, country } \\
\text { remoteness }\end{array}$ & $\begin{array}{l}\text { OLS and } \\
\text { Fixed effects } \\
\text { model }\end{array}$ \\
\hline 2001 & Buch and Piazolo & $\begin{array}{c}\text { Investigation of the impact } \\
\text { of EU enlargement }\end{array}$ & $\begin{array}{c}\text { Cross sectional data, } 9 \text { OECD } \\
\text { and their partner countries, } \\
1998\end{array}$ & $\begin{array}{l}\text { Imports and } \\
\text { Exports }\end{array}$ & $\begin{array}{l}\text { GDPs per capita, distance, EU } \\
\text { membership }\end{array}$ & OLS \\
\hline 2001 & Feenstra et al. & $\begin{array}{l}\text { Evaluation of alternative } \\
\text { theories of trade }\end{array}$ & $\begin{array}{l}\text { Cross sectional, } 110 \text { countries, } \\
1970,1975,1980,1985,1990\end{array}$ & Exports & $\begin{array}{c}\text { GDPs, distance, common border, } \\
\text { common language, existence of } \\
\text { FTA, remoteness }\end{array}$ & OLS \\
\hline 2001 & Porojan & $\begin{array}{c}\text { Investigation of the spatial } \\
\text { effects in the gravity } \\
\text { model }\end{array}$ & $\begin{array}{l}\text { Cross sectional, EU-15 and } 7 \\
\text { OECD countries, } 1995\end{array}$ & $\begin{array}{l}\text { Imports and } \\
\text { Exports }\end{array}$ & $\begin{array}{l}\text { GDPs per capita, distance, EU } \\
\text { and NAFTA membership, } \\
\text { contiguity }\end{array}$ & $\begin{array}{l}\text { OLS, spatial } \\
\text { error, spatial } \\
\text { lag, spatial } \\
\text { error \& lag }\end{array}$ \\
\hline 2001 & Sapir & $\begin{array}{l}\text { Investigation of domino } \\
\text { effects in Western } \\
\text { European regional trade }\end{array}$ & $\begin{array}{c}\text { Cross sectional, } 16 \text { western } \\
\text { european countries, annual } \\
1960-1992\end{array}$ & Exports & $\begin{array}{c}\text { GDPs, distance, common } \\
\text { language, EU and EFTA } \\
\text { membership }\end{array}$ & OLS \\
\hline 2001 & Soloaga and Winters & $\begin{array}{c}\text { Analysis of regionalism } \\
\text { and trade agreement } \\
\text { effects in trade in the } \\
1990 \mathrm{~s}\end{array}$ & $\begin{array}{c}\text { Cross sectional, } 58 \text { countries, } \\
\text { 1980-1996, analysis per year } \\
\text { and averages }\end{array}$ & $\begin{array}{l}\text { Imports and } \\
\text { Exports }\end{array}$ & $\begin{array}{c}\text { GDP, population, remoteness, } \\
\text { distance, land area, common } \\
\text { border, island, common } \\
\text { language, trade agreement } \\
\text { membership }\end{array}$ & $\begin{array}{l}\text { Tobit, fixed } \\
\text { effects. }\end{array}$ \\
\hline 2002 & Eger & $\begin{array}{l}\text { Econometric view on the } \\
\text { estimation of the gravity } \\
\text { model }\end{array}$ & $\begin{array}{c}\text { Panel data, OECD and } 10 \\
\text { Central-Eastern Europe } \\
\text { countries, 1986-1997 }\end{array}$ & Exports & $\begin{array}{c}\text { GDP, GDP per capita, similarity } \\
\text { in country size, exporter and } \\
\text { importer viability of contracts, } \\
\text { exporter and importer rule of } \\
\text { law, real exchange rate, distance, } \\
\text { common border, common } \\
\text { language }\end{array}$ & $\begin{array}{c}\text { Fixed / } \\
\text { random effects } \\
\text { models }\end{array}$ \\
\hline 2002 & Glick and Rose & $\begin{array}{l}\text { Investigation of currency } \\
\text { union effects to trade }\end{array}$ & $\begin{array}{c}\text { Panel data, } 217 \text { countries, } \\
1948-1997\end{array}$ & Exports & $\begin{array}{l}\text { Currency union, distance, GDP, } \\
\text { GDP per capita, common } \\
\text { language, common border, FTA } \\
\text { existence, country landlocked, } \\
\text { number of islands, land areas, } \\
\text { common colonizer, current } \\
\text { colony, ever colony, same nation }\end{array}$ & $\begin{array}{l}\text { OLS, GLS } \\
\text { fixed effects, } \\
\text { GLS random } \\
\text { effects, } \\
\text { between } \\
\text { estimator }\end{array}$ \\
\hline 2003 & Fukao et al. & $\begin{array}{l}\text { Analysis of trade effects } \\
\text { under NAFTA }\end{array}$ & $\begin{array}{c}\text { Panel data, NAFTA members, } \\
1992-1998\end{array}$ & Imports & $\begin{array}{l}\text { GDP per capita, tariffs, total } \\
\text { commodity exports, country } \\
\text { specific factors }\end{array}$ & $\begin{array}{l}\text { OLS with } \\
\text { fixed effects }\end{array}$ \\
\hline
\end{tabular}




\begin{tabular}{|c|c|c|c|c|c|c|}
\hline Year & Authors & Objective & Dataset & $\begin{array}{l}\text { Dependent } \\
\text { Variables }\end{array}$ & $\begin{array}{l}\text { Explanatory } \\
\text { Variables }\end{array}$ & $\begin{array}{l}\text { Estimation } \\
\text { Technique }\end{array}$ \\
\hline 2003 & Baltagi et al. & $\begin{array}{l}\text { Development of a } \\
\text { generalized trade flow } \\
\text { model }\end{array}$ & $\begin{array}{l}\text { Panel data, EU-15, USA, } \\
\text { Japan, 1986-1997 }\end{array}$ & Exports & $\begin{array}{l}\text { GDP, GDP per capita, similarity } \\
\text { in country size, distance }\end{array}$ & $\begin{array}{c}\text { OLS with two } \\
\text { way fixed } \\
\text { effects }\end{array}$ \\
\hline 2003 & Kangas and Niskanen & $\begin{array}{c}\text { Trade in forest products in } \\
\text { EU and Central and } \\
\text { Eastern Europe }\end{array}$ & $\begin{array}{c}\text { Cross sectional data, EU-15 } \\
\text { and } 10 \text { accession countries, } \\
1998\end{array}$ & Exports & $\begin{array}{l}\text { GDP, GDP per capita, distance, } \\
\text { common border, flow between } \\
\text { an EU and accession country }\end{array}$ & OLS \\
\hline 2003 & Filippini and Molini & $\begin{array}{l}\text { Analysis of east Asian } \\
\text { trade flows }\end{array}$ & $\begin{array}{l}\text { Panel data, } 11 \text { EY countries, } \\
\text { USA, Japan, China, } 6 \text { Asian } \\
\text { and } 6 \text { Latin America } \\
\text { countries, 1970-2000 }\end{array}$ & Exports & $\begin{array}{l}\text { Past exports, GDP, population, } \\
\text { distance, technological } \\
\text { differences, region }\end{array}$ & $\begin{array}{l}\text { OLS with } \\
\text { fixed effects }\end{array}$ \\
\hline 2003 & Kurihura & $\begin{array}{c}\text { Impacts of trade flows by } \\
\text { APEC }\end{array}$ & $\begin{array}{c}\text { Panel data, 17 APEC } \\
\text { countries (out of 21), 1980, } \\
\text { 1985, 1990, 1995, 1998 }\end{array}$ & Exports & $\begin{array}{l}\text { Past exports, exchange rate, } \\
\text { GDP, GDP per capita, distance, } \\
\text { common language, common } \\
\text { border, FTA, political union } \\
\text { membership, colony - colonizer }\end{array}$ & OLS, \\
\hline 2003 & Wilson et al. & $\begin{array}{c}\text { Investigation of trade } \\
\text { facilitation and trade flows }\end{array}$ & $\begin{array}{c}\text { Panel data, APEC countries, } \\
1989-2000\end{array}$ & Exports & $\begin{array}{l}\text { GDP, GDP per capita, distance, } \\
\text { NAFTA, ASEAN, LAIA } \\
\text { membership, language (English, } \\
\text { Spanish, Chinese), common } \\
\text { border, tariff, port efficiency, } \\
\text { customs environment, regulatory } \\
\text { environment, e-business }\end{array}$ & $\begin{array}{c}\text { OLS with two } \\
\text { way fixed } \\
\text { effects }\end{array}$ \\
\hline 2003 & Egger and Pfaffermayr & $\begin{array}{c}\text { Investigation of a proper } \\
\text { specification of the gravity } \\
\text { equation using two way } \\
\text { fixed effects }\end{array}$ & $\begin{array}{l}\text { Panel Data, 11 APEP } \\
\text { countries, 1982-1998 }\end{array}$ & Exports & $\begin{array}{l}\text { GDP, population, foreign } \\
\text { currency reserves, exchange rate, } \\
\text { distance, common border, } \\
\text { common language }\end{array}$ & $\begin{array}{c}\text { OLS, two way } \\
\text { fixed effects } \\
\text { model. }\end{array}$ \\
\hline 2004 & Egger & $\begin{array}{l}\text { Estimation of regional } \\
\text { trade bloc effects }\end{array}$ & $\begin{array}{c}\text { Panel data, OECD countries, } \\
1986-1997\end{array}$ & Exports & $\begin{array}{l}\text { GDP, similarity, capital - labor } \\
\text { ratio, high and low skilled labor } \\
\text { ratio to transportation costs, } \\
\text { exporter and importer viability } \\
\text { of contracts, exporter and } \\
\text { importer rule of law, EU, EFTA } \\
\text { and NAFTA membership }\end{array}$ & $\begin{array}{l}\text { Two way } \\
\text { fixed effects - } \\
\text { two way } \\
\text { random effects }\end{array}$ \\
\hline 2004 & $\begin{array}{l}\text { Gopinath and } \\
\text { Echeverria }\end{array}$ & $\begin{array}{l}\text { Effects in the Foreign } \\
\text { direct investment - trade } \\
\text { relationship }\end{array}$ & $\begin{array}{c}\text { Panel data, six countries, } \\
\text { 1989-1998 }\end{array}$ & $\begin{array}{l}\text { Trade to FDI } \\
\text { ratio }\end{array}$ & $\begin{array}{c}\text { GDP, GDP per capita, } \\
\text { population, distance, } \\
\text { accountability, EU membership }\end{array}$ & $\begin{array}{l}\text { OLS with } \\
\text { fixed effects }\end{array}$ \\
\hline 2004 & Pelletiere and Reinert & $\begin{array}{c}\text { Investigation of used } \\
\text { automobile protection and } \\
\text { trade }\end{array}$ & $\begin{array}{l}\text { Panel data, US and } 113 \\
\text { countries, } 1998-2000 .\end{array}$ & Exports & $\begin{array}{l}\text { GDP, population, distance, left } \\
\text { side driving pattern, protection } \\
\text { measure, average tariffs for new } \\
\text { and used cars, region }\end{array}$ & OLS \\
\hline 2004 & Longo and Senkat & $\begin{array}{c}\text { Investigation of the } \\
\text { expansion of Intra African } \\
\text { trade }\end{array}$ & $\begin{array}{c}\text { Panel data, } 41 \text { African and } 15 \\
\text { industrial countries, 1988- } \\
1997\end{array}$ & Exports & $\begin{array}{l}\text { GDP, GDP per capita, country } \\
\text { surface area, common border, } \\
\text { distance, landlocked country, } \\
\text { road length per capita, } \\
\text { telephones per capita, internal } \\
\text { political tension indicators, oil } \\
\text { exporting, FTA participation }\end{array}$ & OLS, TOBIT \\
\hline 2004 & Robers & $\begin{array}{l}\text { Analysis of the proposed } \\
\text { China- Asean FTA }\end{array}$ & $\begin{array}{l}\text { Cross sectional, China and } \\
\text { Asean Countries, } 1996\end{array}$ & Exports & $\begin{array}{c}\text { GDP, GDP per capita, distance, } \\
\text { FTA }\end{array}$ & OLS \\
\hline 2005 & Augier et al. & $\begin{array}{c}\text { Investigation of the } \\
\text { impacts of rules-of-origin }\end{array}$ & $\begin{array}{c}\text { Cross sectional, } 38 \text { countries } \\
\text { (EU and partners), total of } \\
1992-1995\end{array}$ & Exports & $\begin{array}{c}\text { GDP, population, distance, FTA } \\
\text { membership, EU membership, } \\
\text { other country, common border, } \\
\text { common language, cumulation } \\
\text { impact, }\end{array}$ & Fixed effects \\
\hline 2005 & Musila & $\begin{array}{c}\text { Examination of the } \\
\text { intensity of trade creation } \\
\text { and diversion in } \\
\text { COMESA, ECCAS and } \\
\text { ECOWAS }\end{array}$ & $\begin{array}{l}\text { Cross sectional data, } 20 \\
\text { African countries, 1991-1998 }\end{array}$ & Exports & $\begin{array}{c}\text { GDP, population, distance, } \\
\text { common border, common } \\
\text { language, CFA Francophone } \\
\text { zone, Intra COMESA, ECCAS, } \\
\text { ECOWAS trade, Extra } \\
\text { COMESA, ECCAS, ECOWAS } \\
\text { exports and imports }\end{array}$ & OLS \\
\hline
\end{tabular}




\begin{tabular}{|c|c|c|c|c|c|c|}
\hline Year & Authors & Objective & Dataset & $\begin{array}{l}\text { Dependent } \\
\text { Variables }\end{array}$ & $\begin{array}{l}\text { Explanatory } \\
\text { Variables }\end{array}$ & $\begin{array}{l}\text { Estimation } \\
\text { Technique }\end{array}$ \\
\hline 2005 & Sohn & $\begin{array}{l}\text { Analysis of South Korea's } \\
\text { trade floes }\end{array}$ & $\begin{array}{c}\text { Cross sectional. Korea and } 30 \\
\text { trading partners, } 1995\end{array}$ & $\begin{array}{c}\text { Bilateral trade } \\
\text { flows }\end{array}$ & $\begin{array}{c}\text { GDP, GDP per capita, distance, } \\
\text { trade complementarity, APEC } \\
\text { membership }\end{array}$ & OLS \\
\hline 2005 & $\begin{array}{c}\text { Martinez - Zarzoso } \\
\text { and Suarez - Burguet }\end{array}$ & $\begin{array}{l}\text { Investigation of the } \\
\text { relationship between trade } \\
\text { flows and transport cost }\end{array}$ & $\begin{array}{l}\text { EU and five Latin America } \\
\text { countries }\end{array}$ & Imports/Exports & $\begin{array}{c}\text { GDP, GDP per capita, } \\
\text { transportation cost as a function } \\
\text { of weight to value ratio, } \\
\text { distance, volume of imports or } \\
\text { exports, landlocked country, } \\
\text { language, transportation and port } \\
\text { infrastructure characteristics. }\end{array}$ & $\begin{array}{l}\text { OLS with } \\
\text { fixed effects }\end{array}$ \\
\hline 2005 & Paas and Tafenau & $\begin{array}{l}\text { Investigation of trade } \\
\text { flows for countries } \\
\text { involved in the EU } \\
\text { eastwards enlargement } \\
\text { process. }\end{array}$ & $\begin{array}{l}\text { Panel Data, EU-25, 1993- } \\
2002\end{array}$ & Exports & \begin{tabular}{|} 
Population, GDP, distance, EU- \\
15 membership, post-socialist \\
accession countries, land border \\
existence, Baltic sea country, \\
Central European country, \\
Mediterranean country
\end{tabular} & OLS \\
\hline 2005 & Tang & $\begin{array}{l}\text { Analysis of regional } \\
\text { trading arrangements for } \\
\text { the NAFTA, ANZCER } \\
\text { and ASEAN countries }\end{array}$ & $\begin{array}{c}\text { Panel data, } 21 \text { NAFTA, } \\
\text { ANZCER, ASEAN and non- } \\
\text { member countries, 1989-2000 }\end{array}$ & Exports & $\begin{array}{c}\text { GDP, GDP per capita, distance, } \\
\text { volality of exchange rate, income } \\
\text { similarity, developed/developing } \\
\text { country, NAFTA membership } \\
\text { for both or one partner, } \\
\text { ANZCER membership for both } \\
\text { or one partner, ASEAN } \\
\text { membership for both or one } \\
\text { partner, }\end{array}$ & OLS, 2SLS \\
\hline 2005 & Thorpe and Zhang & $\begin{array}{l}\text { Investigation of the } \\
\text { development of intra- } \\
\text { industry trade (IIT) }\end{array}$ & $\begin{array}{l}\text { Panel Data, East Asian } \\
\text { Economies, 1970-1996. }\end{array}$ & $\begin{array}{l}\text { Index of intra- } \\
\text { industry trade } \\
\text { (function of } \\
\text { imports and } \\
\text { exports) }\end{array}$ & $\begin{array}{l}\text { GDP, differences in per capita } \\
\text { income, distance, bilateral } \\
\text { exchange rate, trade orientation, } \\
\text { trade imbalance, economies of } \\
\text { scale. }\end{array}$ & OLS \\
\hline 2005 & Peridy & $\begin{array}{c}\text { Analysis of the AGADIR } \\
\text { FTA effects }\end{array}$ & $\begin{array}{c}\text { Panel data, } 5 \text { MENA and } 42 \\
\text { main import partners, } 1975 \text { - } \\
2001\end{array}$ & Exports & $\begin{array}{l}\text { GDPs, distance, FTA, common } \\
\text { border, common language, trade } \\
\text { complementarity }\end{array}$ & $\begin{array}{l}\text { OLS with two } \\
\text { way random } \\
\text { effects }\end{array}$ \\
\hline 2005 & Kandogan & $\begin{array}{l}\text { Examination of the } \\
\text { Natural Trade Partners } \\
\text { Theory for the Euro- } \\
\text { Mediterranean Region }\end{array}$ & $\begin{array}{c}\text { Cross sectional, EU countries, } \\
1999,2000\end{array}$ & Imports & $\begin{array}{l}\text { GDP, distance, per capita GDP, } \\
\text { real exchange rates, foreign } \\
\text { currency reserves, similarity in } \\
\text { economic sizes, relative factor } \\
\text { endowments }\end{array}$ & $\begin{array}{l}\text { OLS with } \\
\text { fixed effects }\end{array}$ \\
\hline 2005 & Péridy & $\begin{array}{l}\text { Investigation of EMFTA } \\
\text { effects to trade. }\end{array}$ & $\begin{array}{l}\text { Panel Data, Mediterranean } \\
\text { countries with } 42 \text { partners, } \\
\text { 1975-2001 }\end{array}$ & Exports & $\begin{array}{l}\text { GDP, per capita GDP, country } \\
\text { similarity in size, distance, } \\
\text { border type, regional } \\
\text { arrangement between EU and } \\
\text { Mediterranean countries, } \\
\text { language }\end{array}$ & $\begin{array}{l}\text { OLS, Fixed } \\
\text { effects, } \\
\text { Random } \\
\text { Effects }\end{array}$ \\
\hline 2006 & $\begin{array}{l}\text { Antonucci and } \\
\text { Manzocchi }\end{array}$ & $\begin{array}{c}\text { Analysis of the special } \\
\text { trade relation between EU } \\
\text { and Turkey }\end{array}$ & $\begin{array}{c}\text { Panel data, Turkey and trading } \\
\text { partners, } 1967-2001 .\end{array}$ & Exports & $\begin{array}{c}\text { GDP, measure of similarity } \\
\text { between countries, relative factor } \\
\text { endowments, EU membership, } \\
\text { evolving EU relationship, } \\
\text { existence of trade agreements, } \\
\text { distance, border type (sea, land), } \\
\text { specific features of trade } \\
\text { partnerships }\end{array}$ & $\begin{array}{l}\text { GLS with } \\
\text { fixed effects. }\end{array}$ \\
\hline 2006 & Carrère & $\begin{array}{c}\text { Investigation of the effects } \\
\text { of regional trade } \\
\text { agreements }\end{array}$ & $\begin{array}{c}\text { Panel data, } 130 \text { countries, } \\
1962-1996\end{array}$ & Exports & $\begin{array}{c}\text { GDP, GDP per capita, } \\
\text { population, distance, shared } \\
\text { borders, landlocked country, } \\
\text { level of infrastructure, exchange } \\
\text { rates, dummies for FTAs }\end{array}$ & $\begin{array}{l}\text { OLS with two } \\
\text { way random } \\
\text { effects }\end{array}$ \\
\hline 2006 & Kucera and Sarna & $\begin{array}{l}\text { Evaluation of trade union } \\
\text { rights and democracy } \\
\text { effects in exports }\end{array}$ & $\begin{array}{l}\text { Cross sectional, } 162 \text { countries, } \\
\text { averages for period 1993-1999 }\end{array}$ & Exports & $\begin{array}{l}\text { GDP per capita, population, } \\
\text { distance, country surface area, } \\
\text { common border, country } \\
\text { landlocked, island, FTA, } \\
\text { exchange rate }\end{array}$ & $\begin{array}{l}\text { OLS, TOBIT, } \\
\text { WLS }\end{array}$ \\
\hline
\end{tabular}




\begin{tabular}{|c|c|c|c|c|c|c|}
\hline Year & Authors & Objective & Dataset & $\begin{array}{l}\text { Dependent } \\
\text { Variables }\end{array}$ & $\begin{array}{l}\text { Explanatory } \\
\text { Variables }\end{array}$ & $\begin{array}{l}\text { Estimation } \\
\text { Technique }\end{array}$ \\
\hline 2006 & Kang and Fratianni & $\begin{array}{c}\text { Investigation of the effects } \\
\text { of OECD membership and } \\
\text { Religion in trade flows }\end{array}$ & $\begin{array}{l}\text { Panel Data, OECD and non- } \\
\text { OECD countries, } 1980-2003\end{array}$ & Exports & $\begin{array}{l}\text { GDP, GDP per capita, region, } \\
\text { common currency, distance, } \\
\text { common border, common } \\
\text { language, common colonizer, } \\
\text { colonian relationship, OECD } \\
\text { membership }\end{array}$ & OLS \\
\hline 2006 & Baier and Bergstrad & $\begin{array}{l}\text { Examination of FTA } \\
\text { effects }\end{array}$ & $\begin{array}{l}\text { Panel data for years } \\
\text { 1960,1965,.., 2000, } 96 \\
\text { trading partners }\end{array}$ & Bilateral Flows & $\begin{array}{c}\text { GDP, distance, common border, } \\
\text { common language, FTA } \\
\text { membership }\end{array}$ & $\begin{array}{l}\text { OLS, fixed } \\
\text { effects, two- } \\
\text { way fixed } \\
\text { effects, } \\
\text { random } \\
\text { effects, } \\
\text { differentiated } \\
\text { estimates }\end{array}$ \\
\hline 2007 & Elliot & $\begin{array}{c}\text { Analysis of trade flows in } \\
\text { the Caribbean sea. }\end{array}$ & \begin{tabular}{|} 
Panel data, Barbados, \\
Jamaica, Trinidad and \\
Tobago, 1968-2001 and 1969- \\
2003 .
\end{tabular} & $\begin{array}{l}\text { Imports, } \\
\text { exports }\end{array}$ & $\begin{array}{l}\text { Population, distance, } \\
\text { membership in CARICOM } \\
\text { market union. }\end{array}$ & OLS \\
\hline 2007 & Abedini and Peridy & $\begin{array}{l}\text { Analysis of the GAFTA } \\
\text { agreement effects }\end{array}$ & $\begin{array}{c}\text { Panel data, 15GAFTA } \\
\text { countries, 8 GAFTA } \\
\text { candidate countries, another } \\
35 \text { reference countries, 1985- } \\
2000\end{array}$ & Exports & $\begin{array}{c}\text { GDP, distance, common } \\
\text { language, multilateral trade } \\
\text { resistance, information costs, } \\
\text { common border, FTA } \\
\text { participation (EU, NAFTA, } \\
\text { GAFTA etc) }\end{array}$ & $\begin{array}{l}\text { Fixed effects, } \\
\text { random } \\
\text { effects, HTM, } \\
\text { ABB }\end{array}$ \\
\hline 2007 & Kalirajan & $\begin{array}{c}\text { Investigation of regional } \\
\text { cooperation effects in } \\
\text { trade }\end{array}$ & $\begin{array}{c}\text { Panel data, Australia and IOR- } \\
\text { ARC members, 1992-1996 } \\
\text { and 1999-2002 }\end{array}$ & Exports & $\begin{array}{c}\text { GDP, GDP per capita, } \\
\text { population, distance, APEC } \\
\text { membership }\end{array}$ & GLS \\
\hline 2007 & Lee and Park & $\begin{array}{c}\text { Investigation of optimized } \\
\text { regional trade agreements } \\
\text { for east Asia }\end{array}$ & $\begin{array}{c}\text { Panel data, } 50 \text { countries, } \\
\text { 1994-1999 }\end{array}$ & $\begin{array}{l}\text { Bilateral trade } \\
\text { flows }\end{array}$ & $\begin{array}{l}\text { GDP, GDP per capita, distance, } \\
\text { country surface area, common } \\
\text { border, common language, } \\
\text { common colonizer, colony (past } \\
\text { or present), participation in } \\
\text { currency union, tariff, trade } \\
\text { facilitation, FTA membership }\end{array}$ & $\begin{array}{l}\text { OLS with } \\
\text { fixed/random } \\
\text { effects }\end{array}$ \\
\hline 2007 & Bunt and Klaasen & $\begin{array}{l}\text { Investigation of Euro } \\
\text { Effects in trade }\end{array}$ & $\begin{array}{l}\text { Panel data, EU-15, Norway, } \\
\text { Switzerland, Canada, Japan, } \\
\text { USA, 1967-2002. }\end{array}$ & $\begin{array}{l}\text { Bilateral trade } \\
\text { flows }\end{array}$ & $\begin{array}{l}\text { GDP, GDP per capita, FTA } \\
\text { membership, Euro integration }\end{array}$ & $\begin{array}{l}\text { OLS with } \\
\text { fixed effects, } \\
\text { DOLS }\end{array}$ \\
\hline 2007 & $\begin{array}{l}\text { Iwanow and } \\
\text { Kirkpatrick }\end{array}$ & $\begin{array}{l}\text { investigation of trade } \\
\text { facilitation, regulatory } \\
\text { quality and export } \\
\text { performance }\end{array}$ & $\begin{array}{c}\text { Panel data, } 78 \text { countries, } \\
\text { 2000-2004 }\end{array}$ & Exports & $\begin{array}{c}\text { GDP, GDP per capita, } \\
\text { population, distance, remoteness, } \\
\text { tariff, common language, colony } \\
\text { (past/present), common border, } \\
\text { FTA membership, trade } \\
\text { facilitation, quality of regulation, } \\
\text { infrastructure }\end{array}$ & $\begin{array}{c}\text { GLS with } \\
\text { fixed effects }\end{array}$ \\
\hline 2007 & Nowak-Lehmann et al. & $\begin{array}{c}\text { Analysis of customs union } \\
\text { between EU and Turkey }\end{array}$ & $\begin{array}{c}\text { Panel data, Turkey and } 10 \mathrm{EU} \\
\text { countries, } 1998-2002\end{array}$ & Exports & $\begin{array}{l}\text { GDP, GDP per capita, exchange } \\
\text { rate, transport costs }\end{array}$ & $\begin{array}{l}\text { OLS with } \\
\text { fixed effects }\end{array}$ \\
\hline 2007 & Papazoglou & $\begin{array}{l}\text { Analysis of Potential } \\
\text { Trade Flows in Greece.. }\end{array}$ & $\begin{array}{c}\text { Panel of cross-country } \\
\text { data,1993-2003, } 26 \text { countries: } \\
\text { 14 EU members and the } 12 \\
\text { major trading partner } \\
\text { countries. }\end{array}$ & Exports & $\begin{array}{l}\text { GPD, population, distance, EU } \\
\text { membership, common border, } \\
\text { exports of intra-industry type }\end{array}$ & OLS \\
\hline 2007 & $\begin{array}{l}\text { Sarkera, and } \\
\text { Jayasinghe }\end{array}$ & $\begin{array}{l}\text { Analysis of regional trade } \\
\text { agreements and trade in } \\
\text { agri-food products }\end{array}$ & $\begin{array}{l}\text { EU-15 from } 1985 \text { to } 2000,57 \\
\text { countries. }\end{array}$ & $\begin{array}{c}\text { Bilateral trade } \\
\text { flows }\end{array}$ & $\begin{array}{c}\text { Distance, GPD, GPD per capita, } \\
\text { EU (member of the EU), EUO( } \\
\text { degree of openness of the EU } \\
\text { members) }\end{array}$ & OLS \\
\hline 2007 & Tzouvelekas & $\begin{array}{l}\text { Development of a } \\
\text { stochastic coefficient } \\
\text { gravity model }\end{array}$ & 1997, 15 EUcountries. & $\begin{array}{l}\text { Bilateral trade } \\
\text { flows }\end{array}$ & GPD, distance, population & $\begin{array}{c}\text { OLS, } \\
\text { stochastic } \\
\text { varying } \\
\text { coefficient } \\
\text { gravity model }\end{array}$ \\
\hline
\end{tabular}




\begin{tabular}{|c|c|c|c|c|c|c|}
\hline Year & Authors & Objective & Dataset & $\begin{array}{l}\text { Dependent } \\
\text { Variables }\end{array}$ & $\begin{array}{l}\text { Explanatory } \\
\text { Variables }\end{array}$ & $\begin{array}{l}\text { Estimation } \\
\text { Technique }\end{array}$ \\
\hline 2007 & Melitz & $\begin{array}{l}\text { Examination of North - } \\
\text { South Distance }\end{array}$ & $\begin{array}{l}157 \text { Countries, } 1970-1995 \text {, } \\
\text { five year intervals }\end{array}$ & $\begin{array}{l}\text { Bilateral trade } \\
\text { flows }\end{array}$ & $\begin{array}{l}\text { GDP, Distance, common border, } \\
\text { difference North - South, } \\
\text { common language, currency } \\
\text { union, FTA, common country, } \\
\text { ex-colony, common colonizer }\end{array}$ & $\begin{array}{l}\text { OLS fixed } \\
\text { effects }\end{array}$ \\
\hline 2008 & Grant and Lambert & $\begin{array}{l}\text { Investigation of the trade } \\
\text { flow effects of Regional } \\
\text { Trade Agreements } \\
\text { (RTAs). }\end{array}$ & $\begin{array}{c}\text { 1982-2002, AGR and NAGR } \\
\text { commodities. The data set is } \\
\text { derived from the United } \\
\text { Nations Commodity Trade } \\
\text { Statistics Database } \\
\text { (COMTRADE). }\end{array}$ & $\begin{array}{l}\text { Bilateral trade } \\
\text { flows }\end{array}$ & $\begin{array}{l}\text { GDP, Distance, Adjacency, } \\
\text { Language, Landlocked, RTA }\end{array}$ & $\begin{array}{l}\text { OLS fixed } \\
\text { effects }\end{array}$ \\
\hline 2008 & $\begin{array}{c}\text { Bussière, Fidrmuc, and } \\
\text { Schnatz }\end{array}$ & $\begin{array}{c}\text { Analysis of the rapid } \\
\text { trade integration that took } \\
\text { place in the past decade } \\
\text { between the CSEECs and } \\
\text { the euro area. }\end{array}$ & $\begin{array}{c}\text { annual data from } 1980 \text { to } \\
2003,61 \text { countries }\end{array}$ & $\begin{array}{l}\text { Bilateral trade } \\
\text { flows }\end{array}$ & $\begin{array}{c}\text { Distance, territory, border, } \\
\text { language, free trade } \\
\text { arrangements: EU, NAFTA, } \\
\text { MERCOSUR, CEFTA, ASEAN }\end{array}$ & $\begin{array}{l}\text { OLS, fixed } \\
\text { effects, } \\
\text { random } \\
\text { effects, } \\
\text { dynamic OLS, } \\
\text { fixed effects } \\
\text { with regional- } \\
\text { specific time } \\
\text { effects }\end{array}$ \\
\hline 2008 & $\begin{array}{l}\text { Henderson and } \\
\text { Millimet }\end{array}$ & $\begin{array}{c}\text { Estimation of gravity } \\
\text { models-in levels and logs- } \\
\text { via non parametric } \\
\text { methods }\end{array}$ & $\begin{array}{c}132 \text { non-industrial countries, } \\
1948-1997\end{array}$ & $\begin{array}{l}\text { Bilateral trade } \\
\text { flows. }\end{array}$ & \begin{tabular}{|c|} 
Distance, Currency Union, \\
Common Language, Regional \\
trade agreement, Adjacent, \\
Number landlocked, Number of \\
islands
\end{tabular} & \\
\hline 2008 & $\begin{array}{l}\text { SoonchanPark and } \\
\text { Innwon Park }\end{array}$ & $\begin{array}{c}\text { Estimation of the } \\
\text { investment creation and } \\
\text { diversion effects of RTAs }\end{array}$ & $\begin{array}{l}\text { OECD's International Direct } \\
\text { Investment Statistics covering } \\
\text { from } 24 \text { OECD countries to } \\
50 \text { host countries for the } \\
\text { period of } 1982-99 .\end{array}$ & FDI & $\begin{array}{l}\text { GDP in pairs, Skill, openness, } \\
\text { reform, RTA/Insiders, } \\
\text { RTA/Outsiders, RTA, } \\
\text { (RTA/Insiders)·Reform, } \\
\text { (RTA/Outsiders) Reform, log of } \\
\text { distance, common land border, } \\
\text { common language, ex- colony- } \\
\text { colonizer }\end{array}$ & $\begin{array}{l}\text { OLS Fixed } \\
\text { effects, } \\
\text { Random } \\
\text { Effects }\end{array}$ \\
\hline 2008 & $\begin{array}{l}\text { Boriss Siliverstovs, } \\
\text { Dieter Schumacher }\end{array}$ & $\begin{array}{l}\text { Comparison of the OLS } \\
\text { approach applied to the } \\
\text { log-linear form of the } \\
\text { gravity model with the } \\
\text { Poisson Quasi Maximum } \\
\text { Likelihood (PQML) } \\
\text { estimation procedure }\end{array}$ & $\begin{array}{l}\text { 1988-1990, } 22 \text { OECD } \\
\text { countries }\end{array}$ & $\begin{array}{l}\text { Bilateral trade } \\
\text { flows }\end{array}$ & $\begin{array}{c}\text { Distance, adjacency, } \\
\text { membership in a preference area: } \\
\text { European Union, European Free } \\
\text { Trade Agreement, Free Trade } \\
\text { Agreement between the USA } \\
\text { and Canada, Asia-Pacific } \\
\text { Economic Co-operation, ties by } \\
\text { language, historical ties. }\end{array}$ & $\begin{array}{l}\text { OLS, Poisson } \\
\text { Quasi } \\
\text { Maximum } \\
\text { Likelihood } \\
\text { (PQML) }\end{array}$ \\
\hline 2008 & Lampe & $\begin{array}{l}\text { Investigation of bilateral } \\
\text { trade flows in Europe }\end{array}$ & $1857-1875$ & Imports & $\begin{array}{l}\text { National incomes of importer } \\
\text { and exporter, distance, common } \\
\text { border, American Civil War, } \\
\text { tariff levels. }\end{array}$ & $\begin{array}{l}\text { OLS Core, } \\
\text { OLS } \\
\text { Extended, } \\
\text { GLS Core, } \\
\text { GLS } \\
\text { Extended, } \\
\text { PPML Core, } \\
\text { PPML } \\
\text { extended }\end{array}$ \\
\hline 2009 & Kepaptsoglou et al. & $\begin{array}{l}\text { Analysis of the EMFTA } \\
\text { trade agreement }\end{array}$ & $\begin{array}{c}\text { Panel data, EU and } \\
\text { Mediterranean countries, } \\
1993-2007\end{array}$ & $\begin{array}{l}\text { Bilateral trade } \\
\text { flows }\end{array}$ & $\begin{array}{l}\text { Exports and imports, } \\
\text { transportation costs, free trade } \\
\text { agreements, tariffs }\end{array}$ & $\begin{array}{c}\text { SURE with } \\
\text { two way fixed } \\
\text { and random } \\
\text { effects. }\end{array}$ \\
\hline 2009 & Baier and Bergstrand & $\begin{array}{c}\text { Analysis of a simple } \\
\text { method for approximating } \\
\text { international trade-cost } \\
\text { effects using the gravity } \\
\text { equation }\end{array}$ & & & & \\
\hline
\end{tabular}

through trade complementarity. Both studies proposed indices that relate imports and exports for country pairs: Sohn [33] introduced the trade conformity index (TCI), which reflected complementatiry in export and import shares between countries, and Peridy [53] used the index of trade position (ITP) which indicated market share within a trade 
agreement as compared to the rest of the world. Additionally, Thorpe and Zhang [86] used trade imbalance for a country as the difference of imports and exports for a country.

Impedance factors include all those elements that affect trade flows in a negative or positive manner. Transportation costs are the main resistance factors; these include actual freight transportation costs, tariffs, quality of infrastructure etc [71]. Typically, these are approximated by the total distance between the countries' economic centers; that is, the great circle distance calculated by the longitudes and latitudes of centers. Nitsch [65] however proposed a more detailed method for calculating intra-country distances as a function of country size. Nowak-Lehmann et al. [58] on the other hand considered only maritime transportation costs and therefore distances and notes that these are considerably larger compared to land transportation costs. Some studies considered tariffs explicitly [37, 43, 45, 60].

An analysis of transportation costs in trade flows is offered by Martinez-Zarzoso and Suarez-Burguet [71]; they estimated a transport cost equation and include it in their gravity model specification. Their equation involves importer, exporter and commodity type; their variables are distance, weight-to-value-ratio, volume of imports, the case of landlocked countries and common language and infrastructure indices. The authors indicated that distance and poor infrastructure notably increase transportation costs. Kepaptsoglou et al. [64] used a detailed pre-process model for estimating transportation costs between EU and Mediterranean countries. Their model calculated relevant costs for different modes which are then used for estimating the gravity model parameters.

Remoteness is another impedance measure included in gravity model specifications; it indicates the geographic position of countries along markets and has been used in some studies [65, 74, 87]. Originally introduced by Deardorff [24], remoteness is defined as the GDP weighted negative of distance between countries. Moreover, other studies considered exchange rates between partners, since volatility of these rates through time are expected to influence trade flows; Rose [40], Argyriou [31], Eger [76], Thorpe and Zhang [86], Kandogan [55], Carrere [49] and Kucera and Sarna [73] are such cases.

Performance and quality of border and "behind the border" services were among those elements affecting transportation costs. Wilson et al. [45] introduced in the gravity model specification appropriate indicators for including port efficiency, customs environment, e-business existence and the countries' regulatory environments. Longo and Senkat [47] on the other hand, incorporated variables such as road length and telephones per capita, along with indicators of intra-country political tensions. Similarly, Filippini and Molini [19] introduced the notion of technological distance between partners, based on the technological index (TI) proposed by Archibugi and Cogo [88]; this indicator incorporated technology creation and development and diffusion of human resources for each trading partner.
Apart from costs, a variety of impedance factors have been incorporated in the different gravity model specifications, aiming at examining potential barriers to trade flows. Typically encountered factors of that kind are common language, border adjustency and landlocked country, since it is expected that these factors may or may not promote trade between countries. Participation in a customs unions and a trade agreement is a factor frequently found in gravity model specifications; effects of these agreements have been of particular interest during the last decade. These are typically handled as dummy variables, taking a value of 1 when two partners are members of the same union or agreement and 0 otherwise. While use of such dummy variables has been criticized [89], since they can incorporate the effects of other, unrelated factors, they constitute a common approach for analyzing the impacts of regional agreements etc. Example cases include membership in customs and monetary unions, like EEC and EU, or trade agreements such as EMFTA, NAFTA, AGADIR, ASEAN and so on.

Similarly, sharing the same currency, being part of the same nation, colonies in the past or present, or even having a common colonizer, are factors expected to affect trade flows between regions, with their potential impacts also being captured through dummy variables in various studies.

\subsection{Methodological Aspects}

The Ordinary Least Squares (OLS) method has been traditionally the usual technique for estimating the coefficients of the gravity model specification in its loglinear form. While OLS is still implemented for analyzing and evaluating policies [81], researchers have indicated methodological and modeling flaws in the development of gravity models using OLS. As noted by Henderson and Millimet [80], in most empirical studies, implementation assumptions are not in line with the underlying theoretical models. Theoretical considerations on the proper use and deviations from the gravity model have been a topic of considerable interest in the literature (examples include Feenstra et al. [87]; Anderson and van Wincoop [28]; Henderson and Millimet [80]).

Of particular interest however, is the seminal work of Anderson and van Wincoop [28] who refined the theoretical foundations of the gravity models to properly account for the endogeneity of trade costs and the consideration of institutional barriers to trade. Based on a theoretical model of trade they indicated that costs of bilateral trade between two regions are affected by the average trade cost of each region with the rest of its trading partners (the so-called lack of multilateral resistance ${ }^{1}$ ) and provided evidence of border effects in trade, using a Non-linear least squares (NLS) model. According to Baier and Bergstrand [82], an alternative yet simpler way of treating multilateral resistance is the use of a fixed effect model. Indeed, since Anderson and Van Wincoop, most relevant studies have employed fixed effects techniques for developing gravity models. In

\footnotetext{
${ }^{1}$ Multilateral resistance is the average barrier between two partners to trade with others.
} 
2009, aiming at bridging the gap between theory and practice as posed by Anderson and van Wincoop [28], Baier and Bergstrad [82] proposed an approximation of multilateral effects to be used with OLS; they reported that their method provided comparable results both to Anderson and van Wincoop [28] and fixed effects approaches. In another study by Baier and Bergstrad [5], the endogeneity of FTAs was also considered; they again proposed a fixed and random effects specification to account for this.

From a practical perspective, treatment of interaction effects is a necessity in order to avoid biases and inconsistent estimates [76]. For example, Baltagi et al. [77] concluded that a lack of interaction effects may lead to biased results and incorrect inferences in the gravity model and highlighted the importance of controlling for all interactions in both the cross-sectional and the temporal dimensions. In this sense, use of two way fixed effects models has been recognized as potentially useful to capture cross-country and time interactions. According to Egger [76], selection between fixed and random effects models depends on the interests of the analysis, the country sample, the data properties and the underlying theoretical model used. In general, most empirical studies indicate that fixed effects models tend to provide better results; Egger [76] reported that two way fixed or random effect models are needed to capture crosscountry and time effects, but notes that random effects models should be considered if they are adequately consistent and there is an interest in estimating timeinvariant effects [90]; otherwise, fixed effects models are the only available alternative.

Glick and Rose [42] implemented fixed and random effects models; they reported that their fixed effects model provides robust results. Egger and Pifaffermayr [78] argued on the necessity of incorporating two way fixed effects and Wilson et al. [45] reported the robustness of a two-way fixed effects model with respect to invariant factors (such as tariffs). Antonucci and Manzocchi [91] again preferred a fixed effects model from a random effects model using a two step approach: first, they perform a fixed effects model regression and then a cross-section regression with country specific effects as the dependent variable is run. Indeed, most recent studies prefer fixed effects as their panel correction technique. Filippini and Molini [19] also used a fixed effect model, assuming that heterogeneity is correlated with the regressors and note that long-run data allows them to disregard any endogeneity problems and apply the fixed effect model without any bias.

Among other efforts, Kalijaran [92] introduced a stochastic coefficient gravity model; a model fitting in the same category was implemented almost a decade later by Tzouvelekas [79]. Porojan [4] exploited spatial econometrics in an effort to capture the model's spatial effects. Egger [46] adopted a three stage modeling approach for examining trade effects with the use of a gravity model; he sequentially estimated a "fixed effects", an instrumental variable and a "random effects" model. Peridy [53] used a random effects model; he applied several related estimators (HTM, dynamic $\mathrm{ABB})$ and notes that these estimators are superior compared to the standard random effects estimator for the case of the gravity model specification analyzed. Carrere [49] argued that modeling of bilateral effects as random variables is more appropriate compared to fixed effects and considered the instrumental Hausman - Taylor estimation for that purpose. In another study, Peridy [54] exploited a variety of modeling techniques (OLS, fixed effects, random effects, HTM, GMM and $\mathrm{ABB}$ ) and comes up with a number of comments by comparing their results; with respect to static models, he found that all models have "significant and similar" parameters, while GMM is the most appropriate dynamic model. Henderson and Millimet [80] proposed a nonparametric technique for estimating the gravity equation and report promising results. Novak-Lehmann et al. [58] implemented a seemingly unrelated regression (SUR) technique, controlling for cross country fixed effects while Kepaptsoglou et al. [64] used the same technique, considering two-way fixed effects. Finally, Baier and Bergstrad [82] proposed an approximation of multilateral effects to be used with OLS; they reported that their method provided comparable results both to Anderson and van Wincoop [28] and fixed effects approaches.

From a different perspective, a couple of recent studies by Santos Silva and Tenreyro [93] and Sillverstovs and Schumacher [81] criticized the original log-linear transformation for estimating the gravity model coefficients. Santos Silva and Tenreyro [93] reported that the consistency of the OLS estimator typically used in the log-linear form depends on an unrealistic assumption of the error term and recommended the estimation of the gravity model in its original multiplicative form. To achieve that, they suggested a Poisson quasi maximum likelihood (PQML) technique. Siliverstovs and Schumacher [81] compared the PQMS technique with OLS and found evidence of the former's superiority.

\section{DISCUSSION}

\subsection{Empirical Findings}

With over 55 papers published within the last decade, the gravity model has been established as a major instrument for analyzing trade flows and explaining effects of related trade agreements. Despite earlier criticism, the research community has made efforts both in improving the model's theoretical foundation (see, for example, Anderson and van Wincoop [28]) and adopted novel econometric methods for estimating its parameters with more accuracy.

Among empirical studies, most focus on assessing policies and their implications, particularly the effects of various regional trade agreements; studies on general trade flow analyses on the other hand have been limited during the last decade. Some of these studies present novel econometric approaches while other studies deal with advancing the econometric estimation of the gravity model.

Panel data sets are preferred in recent gravity model studies with only a handful of studies remaining on crosssectional models; advantages of panel data have been exploited by most researchers. As for explanatory variables, a variety of them has been introduced in the gravity model 
specification: GDP, GDP per capita and distance are the most common variables representing demand and supply (mass variables). Similarity is rarely used as an indicator. Among impedance variables, distance is always encountered (as expected); other factors such as common language and border are commonly used as dummy variables. Indicators such as remoteness and trade complementarity are applied in a few cases only. Only a couple of studies tend to analyze transport costs in detail, a result of difficulty in its detailed calculation. Dummy variables are frequently adopted for capturing impacts of trade agreements, custom unions and similar country characteristics (for example same nation, colonizer, language etc), despite criticism by past studies. In general, GDP and population are the most common mass variables (with a few exceptions), while impedance is described by distance and a variety of factors enhancing or discouraging trade.

From a methodological point of view, plain OLS use has been limited, especially after the conclusions drawn by Anderson and van Wincoop [28]. Introduction of interaction effects (bilateral, time or two-way) has been a common practice in recent studies; however, as noted, the selection among random and fixed effects relies on the interests of the analysis, the country sample, the data properties and the underlying theoretical model used. For example, Egger [76] notes that association of estimators with short and long term planning estimators when comparing results. Fixed effects are better for short term prediction purposes. He also states that random effects models should be considered if they are adequately consistent and there is an interest in estimating time-invariant effects. In general though, most empirical studies tend to highlight fixed effects approaches as the most appropriate for estimating the gravity model coefficients.

Apart from the issue of interaction, there has been some discussion on the proper estimators and econometric methods used, as well as on the use of the traditional loglinear form of the model. Some researchers test advanced econometric methods and claim better performance; some case these methods are theory driven while in other cases are a result of past empirical findings. However, the complexity and usability of these methods remains an issue; Baier and Bergstrad [82], for example, indicate the difficulties in using Anderson and van Wincoop [28] method. Similarly, despite evidence on better performance when directly estimating the multiplicative form of the gravity, the traditional form is widely accepted because of its simple form and understandability.

\subsection{Free Trade Agreements}

Trade agreements have been widely analyzed with the use of gravity models. However, Baier and Bergstrad [5] noted that recent studies do not provide clear evidence on the positive effects of these agreements towards creating or diverting trade. Our review of relevant studies leads to a similar conclusion. For example, Endoh [39] found that the Latin American Free Trade Agreement (LAFTA) has exhibited neither trade creation nor trade diversion on trade with Japan and Fukao et al. [43] provided evidence of some trade diversion as a result of the North American Free Trade
Agreement (NAFTA). Soloaga and Winters [41] found only limited evidence of trade diversion because of the EU and EFTA. Roberts [48] investigated the potential of a FTA between China and ASEAN countries; he concluded that neither trade creation nor diversion is expected. Eger [46] indicated that while FTAs are not expected to have a shortterm impact on trade volumes, a considerable long-run trade creation is anticipated; he reported a $15 \%$ long-term increase for NAFTA members. An analysis of trade agreements in African countries (COMESA, ECCAS, ECOWAS) was provided by Musila [50]; the author does not find any considerable impacts in trade diversion and creation Similar conclusions were drawn for the COMESA by Rojid [94] and for the AGADIR agreement by Peridy [53], as a result of the lack of trade complementarity between its member countries.

Tang [52] examined the effects of the NAFTA, ANZCER and ASEAN FTAs. His results showed that trade within member countries has increased, ANZCER FTA has resulted in trade diversion from non-members and ASEAN FTA has led to a trade increase with non-members (something that has not been observed for NAFTA). Peridy [54] investigated trade effects of the Euro-Mediterranean FTA (EMFTA); he reported that the FTA resulted in an increase of Mediterranean countries' exports to the EU by $20 \%-27 \%$, indicating trade creation and accounted for the large EU share of Mediterranean exports. Carrere [49] concluded that “...regional trade agreements generate a significant increase in trade between members, often at the expense of the rest of the world". Abedini and Peridy [95] reported a $20 \%$ increase in trade flows between regions belonging to the GAFTA agreement. Lee and Park [59] proposed new FTAs for East Asia; they noted that trade facilitation would enhance trade creation between FTA members and reduces trade diversion among them. Moreover they indicated that their proposed FTAs would be beneficial compared to existing conditions. Kalijaran [57] reported that Australia was expected to have more gains of its potential exports because of the IOC-ARC agreement and Grant and Lambert [61] indicated that the type and characteristics of trade agreements for agricultural products play a significant role in actually improving trade, while positive effects to trade may not occur immediately. Finally, in their study, Baier and Bergstrad [5] attempted to clarify the effects of FTAs in trade, by exploiting the prevailing theoretical background of the gravity model and modern econometric studies. Their treatment of the FTAs as endogenous variables, subject to interaction effects, led to the conclusion that FTAs do affect trade considerably.

\section{CONCLUSIONS}

This paper critically reviewed and analyzed recent empirical studies exploiting the gravity model in trade flow. Based on its robust performance, the gravity model has been particularly successful and popular among researchers, despite past criticism on its theoretical background. Over 75 papers in the last decade have either used it for analyzing trade policies and their implications or improved its performance; most of the policies examined focused on the effects of FTA agreements. Gravity models developed 
exhibit a wide range of econometric novelties; however, fixed and random effects models are followed by the majority of the research community. Results on FTA performance are still contradictory; some studies indicate trade creation and diversion while others do not, while the potential of treating interaction effects for FTAs may provide a clear view. As noted by Beier and Bergstrad [5], accounting for FTA endogeneity would be an important element of future empirical studies.

\section{REFERENCES}

[1] Timbergen J. Shaping the world economy, New York, NY: Twentieth Century Fund 1962.

[2] Linneman H. An econometric study of world trade flows. Amsterdam: North-Holland Publishing Co. 1966.

[3] Bergstrand $\mathrm{J} \mathrm{H}$. The gravity equation in international trade: some microeconomic foundations and empirical evidence. Rev Econ Stat 1985; 67(3): 474-81.

[4] Porojan A. Trade flows and spatial effects: the gravity model revisited. Open Econ Rev 2001; 12: 265-80.

[5] Baier SL, Bergsrtand H. Do free trade agreements actually increase members' international trade? J Int Econ 2007; 71(1): 72-95.

[6] The World Bank. Global Economic Prospects, Washington DC 2005.

[7] Transek AB, CERUM, INRO, TOI, NEA. Ideas for a new Swedish Freight Model. Oslo, Norway 2001.

[8] Kouparitsas MA. Should trade barriers be phased-out slowly? A case study of North America. J Policy Model 2001; 23(8): 875-900.

[9] Augier P, Gasiorek M. The welfare implications of trade liberalization between the Southern Mediterranean and the EU. Appl Econ 2003; 35(10): 1171-90.

[10] Chisic R. Gradualism in free trade agreements: a theoretical justification. J Int Econ 2003; 59(2): 367-97.

[11] Baier SL, Bergstrand JH. Economic determinants of free trade agreements. J Int Econ 2004; 64(1): 29-63.

[12] Bond EW, Riezman RG, Syropoulos C. A strategic and welfare theoretic analysis of free trade areas. J Int Econ 2004; 64(1): 1-27.

[13] Raff $\mathrm{H}$. Preferential trade agreements and tax competition for foreign direct investment. J Public Econ 2004; 88(12): 2745-63.

[14] Ornelas E. Endogenous free trade agreements and the multilateral trading system. J Int Econ 2005; 67(2): 471-97.

[15] Siriwardana M. The Australia-United States free trade agreement: an economic evaluation. Am N J Econ Finance 2007; 18(1): 11733.

[16] Hertel T, Hummels D, Ivanic M, Keeney R. How confident can we be of CGE-based assessments of free trade agreements? Econ Model 2007; 24(3) : 611-35.

[17] Head K. Gravity for beginners. Canada: University of British Columbia 2003.

[18] Baldwin RE. Towards an integrated Europe. London: Centre for Economic Policy Research 1994.

[19] Filippini C, Molini V. The determinants of East Asian trade flows: a gravity equation approach. J Asian Econ 2003; 14(5): 695-711.

[20] Anderson IE. A theoretical foundation of the gravity equation. Am Econ Rev 1979; 69(1): 106-16.

[21] Krugman P. Scale economies, product differentiation, and the pattern of trade. Am Econ Rev 1979; 70(5): 950-9.

[22] Helpman E, Krugman P. Market structure and foreign trade. Cambridge, MA: MIT Press 1985.

[23] Bergstrand JH. The Hecksher-Ohlin-Samuelson model, the linder hypothesis, and the determinants of bilateral intra-industry trade. Econ J 1990; 100(4): 1216-29.

[24] Deardoff A. Determinants of bilateral trade: does gravity work in a neoclassical world?. In: Frankel JA, Ed. The regionalization of the world economy. Chicago, IL: University of Chicago Press 1998.

[25] Anderson JE, Marcouiller D. Trade, location and security: an empirical investigation. NBER working Paper No 7000, 1999.

[26] Baier SL, Bergstrand JH. The growth of the world trade: tariffs, transport costs, and income similarity. J Int Econ 2001; 53(1): 1-27.

[27] Evenett SJ, Keller W. On theories explaining the success of the gravity equation. J Polit Econ 2002; 110(2): 281-316.

[28] Anderson JE, van Wincoop E. Gravity with gravitas: a solution to the border puzzle. Am Econ Rev 2003; 93(1): 171-92.
[29] Washington S, Karlaftis MG, Mannering FL. Statistical and econometric methods for transportation data analysis. Boca Raton, FL: Chapman \& Hall/CRC Press 2003.

[30] Breuss F, Egger P. How reliable are the estimations of east-west trade potentials based on cross-section gravity analyses? Empirica 1999; 26(2): 81-94.

[31] Arghyrou MG. EU participation and the external trade of Greece: an appraisal of the evidence. Appl Econ 2000; 32(2): 151-9.

[32] Kangas K, Niskanen A. Trade in forest products between European Union and the Central and Eastern European access candidates. Forest Policy Econ 2003; 5(3): 297-304.

[33] Sohn C-H. Does the gravity model explain South Korea's trade flows? Japanese Econ Rev 2005; 56(4): 417-30.

[34] Elliott DR. Caribbean regionalism and the expectation of increased trade: insights from a time-series gravity model. J Int Trade Econ Dev 2007; 16(1): 117-36.

[35] Papazoglou C. Greece's potential trade flows: a gravity model approach. Int Adv Econ Res 2007; 13(4): 403-14.

[36] Lampe M. Bilateral trade flows in Europe, 1857-1875: a new dataset. Res Econ Hist 2008; 26(1): 81-155.

[37] Pelletiere D, Reinert KA. Used automobile protection and trade: Gravity and ordered probit analysis. Empir Econ 2004; 29(4): 73751 .

[38] Sarker R, Jayasinghe S. Regional trade agreements and trade in agri-food products: evidence for the european union from gravity modeling using disaggregated data. Agric Econ 2007; 37(1): 93104.

[39] Endoh M. Trade creation and trade diversion in the EEC, the LAFTA and the CMEA: 1960-1994. Appl Econ 1999; 31(2): $207-$ 16.

[40] Rose AK. Currency unions-one money, one market: the effect of common currencies on trade. Econ Policy 2000; 15(30): 7-45.

[41] Soloaga I, Winters A. Regionalism in the Nineties: What Effect on Trade?" N Am J Econ Finance 2001; 12(1): 1-29.

[42] Glick R, Rose AK. Does a currency union affect trade? The timeseries evidence. Eur Econ Rev 2002; 46(6): 1125-51.

[43] Fukao K, Okubo T, Stern RM. An econometric analysis of trade diversion under NAFTA. N Am J Econ Finance 2003; 14(1): 2-24.

[44] Kurihara Y. APEC: International trade and output". Pac Econ Rev 2003; 8(3): 207-17.

[45] Wilson JS, Mann CL, Otsuki T. Trade facilitation and economic development: a new approach to quantifying the impact. World Bank Econ Rev 2003; 17(3): 367-89.

[46] Egger P. Estimating regional trading bloc effects with panel data. Rev World Econ 2004; 140(1): 151-66.

[47] Longo R, Sekkat K. Economic obstacles to expanding intra-african trade". World Dev 2004; 32(8): 1309-21.

[48] Roberts BA. A gravity study of the proposed China-Asean free trade area. Int Trade J 2004; 18(4): 335-53.

[49] Carrere C. Revisiting the effects of regional trade agreements on trade flows with proper specification of the gravity model. Eur Econ Rev 2006; 50(2): 223-47.

[50] Musila JW. The Intensity of trade creation and trade diversion in COMESA, ECCAS and ECOWAS: a comparative analysis. J Afr Econ 2005; 14(1): 117-41.

[51] Paas T, Tafenau E. Regional trade clusters in promoting eastward enlargement of European union. Trans Stud Rev 2005; 12(1): 7790 .

[52] Tang D. Effects of the regional trading arrangements on trade: evidence from the NAFTA, ANZCER and ASEAN Countries, 1989-2000. J Int Trade Econ Dev 2005; 14(2): 241-65.

[53] Peridy N. The trade effects of the Euro-mediterranean partnership: what are the lessons for ASEAN countries?. J Asian Econ 2005; 16(1): 125-39.

[54] Peridy N. Toward a Pan-Arab free trade area: assessing trade potential effects of the Agadir agreement. Dev Econ 2005; XLIII-3: 329- 45 .

[55] Kandogan Y. Evidence for the natural trade partners theory from the Euro-Mediterranean region. working paper series, No.2005-01, University of Michigan-Flint School of Management, 2005.

[56] Kang H, Fratianni M. International trade, OECD membership, and religion. Open Econ Rev 2006; 17(4-5): 493-508.

[57] Kalirajan K. Regional cooperation and bilateral trade flows: an empirical measurement of resistance. Int Trade J 2007; 21(2): 85107. 
[58] Nowak-Lehmann F, Herzer D, Martinez-Zarzoso I, Vollmer S. The impact of a customs union between turkey and the EU on Turkey's exports to the EU. JCMS. J Common Market S 2007; 45(3): 71943.

[59] Engelbrecht H-J, Pearce C. The GATT/WTO has promoted trade, but only in capital-intensive commodities! Appl Econ 2007; 399(12): 1573-81.

[60] Lee H, Park I. In search of optimized regional trade agreements and applications to East Asia”. World Econ 2007; 30(5): 783-806.

[61] Grant JS, Lambert DM. Do regional trade agreements increase members' agricultural trade?" Am J Agric Econ 2008; 90(3): 76582.

[62] Bhattacharya SK, Bhattacharyay BN. An empirical analysis on prospects and challenges of BIMSTEC-Japan trade intergration. J Asian Econ 2007; 18(3): 509-536

[63] Park I, Park S. Reform creating regional trade agreements and foreign direct investment: applications for East Asia. Pac Econ Rev 2008; 13(5): 550-66.

[64] Kepaptsoglou K, Tsamboulas D, Karlaftis MG, Marzano V. Analyzing free trade agreements effects in the mediterranean region: a sure gravity model based approach. Transp Res Rec 2009; 2097: 88-96

[65] Nitsch V. National borders and international trade: evidence from the European Union. Can J Econ 2000; 33(4): 1091-105.

[66] Buch CM, Piazolo D. Capital and trade flows in Europe and the impact of enlargement. Econ Syst 2001; 25(3): 183-214.

[67] Bun MJG, Klaassen FJGM. The Euro effect on trade is not as large as commonly thought. Oxford B Econ Stat 2007; 69(4): 473-96.

[68] Sapir A. Domino effects in Western European regional trade, 19601992. European J Polit Econ 2001; 17(2): 377-88

[69] Gopinath M, Echeverria R. Does economic development impact the foreign direct investment-trade relationship? a gravity-model approach. Am J Agric Econ 2004; 86(3): 782-7.

[70] Augier P, Gasiorek M, Lai Tong C. The impact of rules of origin on trade flows". Econ Policy 2005; 20(43): 567-624.

[71] Martinez-Zarzoso I, Suarez-Burguet C. Transport costs and trade: empirical evidence for latin american imports from the European union. J Int Trade Econ Dev 2005; 14(3): 353-71.

[72] Egger P. On the role of distance for bilateral trade. World Econ 2008; 31(5): 653-62

[73] Kucera D, Sarna R. Trade union rights, democracy, and exports: a gravity model approach. Rev Int Econ 2006; 14(5): 859-82.

[74] Iwanow T, Kirkpatrick C. Trade facilitation, regulatory, quality and export performance. J Int Dev 2007; 19(6): 735-53.

[75] Melitz J. North, South and distance in the gravity model. Eur Econ Rev 2007; 51(4): 971-991.

[76] Egger P. An econometric view on the estimation of gravity models and the calculation of trade potentials. World Econ 2002; 25(2): 297-312.
[77] Baltagi BH, Egger P, Pfaffermayr M. A generalized design for bilateral trade flow models. Econ Lett 2003; 80(3): 391-7.

[78] Egger P, Pfaffermayr M. The proper panel econometrics specification of the gravity equation: a three-way model with bilateral interaction effects. Empir Econ 2003; 28(3): 571-80.

[79] Tzouvelekas V. Accounting for pairwise heterogeneity in bilateral trade flows: a stochastic varying coefficient gravity model. App Econ Lett 2007; 14(12): 927-30.

[80] Henderson DJ, Millimet DL. Is gravity linear? J Appl Econ 2008, 23(2): 137-72.

[81] Siliverstovs B, Schumacher D. Estimating gravity equations: to log or not to log?. Empir Econ 2008; 36(3): 645-69.

[82] Baier SL, Bergstrand JH. Bonus vetus OLS: a simple method for approximating international trede-cost effects using the gravity equation. J Int Econ 2009; 77(1): 77-85

[83] Mátyás L. Proper econometric specification of the gravity model World Econ 1997; 20(3): 432-4.

[84] Ghosh S, Yamarik S. Are regional trading arrangements trade creating? an application of extreme bounds analysis. J Int Econ 2004; 63(2): 369-95.

[85] Helpman E. "Imperfect competition and international trade: evidence from fourteen industrial countries". J Jpn Int Econ 1987 1(1): $62-81$.

[86] Thorpe M, Zhang Z. Study of the measurement and determinants of intra-industry trade in East Asia”. Asian Econ J 2005; 19(2): 23147.

[87] Feenstra RC, Markusen JR, Rose AK. Using the gravity equation to differentiate among alternative theories of trade. Can J Econ 2001; 34(2): 430-47.

[88] Archibugi D, Cogo A. A new indicator of technological capabilities for developed and developing countries. Mimeo, Italy: Italian National Research Council, 2002.

[89] Polak JJ. Is APEC a natural regional trading bloc?. World Econ 1996; 19(5): 533-43.

[90] Brun J-F, Carrere C, Guillaumont P, de Melo J. Has distance died? evidence from a panel gravity model. World Bank Econ Rev 2005; 19(1): 99-120.

[91] Antonucci D, Manzocchi S. Does Turkey have a special trade relation with the EU? A gravity model approach. Econ Syst 2006, 30(2): 157-69.

[92] Kalirajan K. Stochastic varying coefficients gravity model: an application in trade analysis. J Appl Stat 1999; 26(2): 185-93.

[93] Santos Silva JMC, Tenreyro S. The log of gravity. Rev Econ Stat 2006; 88(4): 641-58

[94] Rojid S. COMESA trade potential: a gravity approach. Appl Econ Lett 2006; 13(14): 947-51.

[95] Abedini J, Peridy N. The greater arab free trade area (GAFTA): an estimation of the trade effects. J Econ Integr 2008; 23(4): 848-72. 